\title{
Scoring Rules over Subsets of Alternatives : Consistency and Paradoxes
}

\author{
Eric Kamwa* \\ Normandie Univ, France. UNICAEN, CREM UMR-CNRS 6211. UFR Sciences Economiques, 19 Rue claude Bloch, \\ F14000 Caen. \\ Vincent Merlin \\ Normandie Univ, France. UNICAEN, CREM UMR-CNRS 6211. UFR Sciences Economiques, 19 Rue claude Bloch, \\ F14000 Caen.
}

\begin{abstract}
We know since the works of Gehrlein and Fishburn (1980, 1981), Fishburn (1981) and Saari $(1987,1988,1990)$ that, the collective rankings of scoring rules are not stable when some alternatives are dropped from the set of alternatives. However, in the literature, attention has been mainly devoted to the relationship between pairwise majority vote and scoring rules rankings. In this paper, we focus on the relationships between four-candidate and three-candidate rankings. More precisely, given a collective ranking over a set of four candidates, we determine under the impartial culture condition, the probability of each of the six possible rankings to occur when one candidate is dropped. As a consequence, we derive from our computations, the likelihood of two paradoxes of committee elections, the Leaving Member Paradox (Staring, 1986) and the Prior Successor Paradox which occur when an elected candidate steps down from a two-member committee.
\end{abstract}

Keywords: Scoring rule, Consistency, Collective Ranking, Committee, Paradox, Impartial Culture.

\section{Introduction}

One of the main objectives of the social choice theory is the aggregation of individual preferences into a collective ranking that is the determination of a complete order over the set of the alternatives or candidates. This objective can be achieved when every voter gives points to each candidate in accordance with his preference order. Hence, the candidate with the highest total score will be ranked at the top of the collective ranking and the one with the lowest score will be at the bottom. The Plurality rule, the Antiplurality rule and the Borda rule are among others, some well known scoring rules that can be used for a such objective. With the Plurality rule, a candidate's score is the number of times he is top ranked in the individual rankings. With the

${ }^{*}$ Corresponding author.

Email addresses: eric.kamwa@unicaen.fr (Eric Kamwa), vincent.merlin@unicaen.fr (Vincent Merlin ) 
Antiplurality rule, a candidate's score is equal to the number of voters that do not rank him last in their rankings. With $m$ candidates, the Borda rule gives $m-j$ points to a candidate each time, he is ranked $j$-th in a voter's ranking. The total number of points received by a candidate, defines his Borda's score.

In this context, some issues have been addressed regarding the stability of a collective ranking over subsets of candidates. How a collective ranking can be altered after one or more candidates are removed from the competition? Is the new collective ranking consistent with the former one?

These issues may also arise in the context of committee elections. Assume that, when electing a committee of size $g$, this committee is made by the candidates with the $g$ greatest scores (the $g$ top ranked candidates of the collective ranking). Staring (1986) pointed out that, when electing committees, if a member of an elected committee leaves, a new ballot, ceteris paribus ${ }^{1}$, could lead to a new committee without some members of the previous ones; even worse, the two committees may be disjoint. This is called the Leaving Member Paradox (LMP) ${ }^{2}$. In this paper, we define another paradox of committee elections which is less severe than the LMP: the Prior Successor Paradox (PSP). Since the elected committee is formed by the candidates with the $g$ greatest scores, we define the Prior Successor as the candidate with the $g+1$-th best score. The PSP occurs if after a member of the elected committee leaves, a new ballot (given the subset of candidates) leads ceteris paribus to a committee containing all the $g-1$ members of the previous committee without the Prior Successor. The LMP and the PSP, as defined, are two non negligible inconsistencies that may tarnish the image of some voting systems. One of the objective of this paper is to study the occurrence of these paradoxes.

The origin of the questions on the stability of a collective ranking over subsets of candidates can be tracked back to the Borda-Condorcet debate. At the end of the 18th century, Borda (1781) and Condorcet (1785) who were members of the Paris Royal Academy of Sciences, proposed alternative voting rules to the one that was in use in the academy (see McLean and Urken (1995)). The Borda rule picks as the winner, the candidate with the highest Borda's score. Condorcet (1785) criticized the Borda rule in that it can exist a candidate that is preferred by more than half of the electorate to the Borda winner. Condorcet (1785) proposed a rule based on pairwise comparisons $^{3}$. According to this rule, a candidate should be declared the winner if he beats all the other candidates in pairwise majority; such a candidate is called the Condorcet winner. Nonetheless, the Condorcet rule has a main drawback : it can lead to majority cycles ${ }^{4}$ in some circumstances. Though the Borda-Condorcet debate ${ }^{5}$ about the choice of the best voting rule is still alive, everyone agrees that they were the first authors who emphasized the fact that scoring rules and pairwise comparisons do not always lead to the same ranking.

The Borda-Condorcet debate can be envisioned in a broader picture : what are the relationships between the rankings on a set of candidates $A$ and the rankings on the subset $B$ included in $A$ for a given preference profile? Apart from classical studies analyzing the relationships between pairwise voting and scoring rules (see Dodgson (1876), Nanson (1882), Smith (1973), Fishburn

\footnotetext{
${ }^{1}$ This means that, voters keep their preferences unchanged on the rest of candidates no matter who is the leaving candidate.

${ }^{2}$ An example illustrating the LMP is provided by Staring (1986) for a voting system under which voters vote for as may candidates as there are vacancies to be filled.

${ }^{3}$ See Young (1988) for a modern interpretation of Condorcet's rule.

${ }^{4}$ Let us take three candidates $a, b$ and $c$ in order to illustrate what a majority cycle is in a simple way. For a given electorate, if $a$ is majority preferred to $b$ and $b$ is majority preferred to $c$ and $c$ is majority preferred to $a$, this describes a majority cycle with three candidates.

${ }^{5}$ Finally, the Borda rule was retained by the Academy.
} 
and Gehrlein (1976)), the first extension of these contributions is due to Fishburn (1981) who showed that there always exist a preference profile for which removing any candidate from $A$ leads to the reversed ranking on the remaining set of candidates. In a seminal paper Saari (1988) generalized this result by studying simultaneously the rankings on all the subsets of $A$ for a given profile. He showed that, for most of the scoring rules (the Borda rule being one of the few exceptions) anything can happen for some profiles and no relationship prevails. This result was further developed in Saari (1987, 1990, 1996).

This result could cast a doubt on the practical use of scoring rules. What remains is to see whether these paradoxical results are just rare oddities or betray a more generalized behavior. In modern social choice theory, many works have tried to analyze the relationships between pairwise and scoring rules. Among others, we can mention the works of Gehrlein and Fishburn (1976), Gehrlein and Fishburn (1980, 1981), Gehrlein et al. (1982), Fishburn (1981), Tataru and Merlin (1997), Van Newenhizen (1992) and more recently, Cervone et al. (2005). Most of them prove that given a scoring rule and a collective ranking, there is no reason to think that the pairwise comparisons will always be consistent with this collective ranking.

However, this line of research barely analyzed anything but pairwise relationships. In threecandidate elections, Gehrlein and Fishburn $(1980,1981)$ computed the limit probabilities under the Impartial Culture (IC) condition (defined later) that, given a scoring rule, the pairwise comparisons between candidates agree with the collective ranking. They showed that, the agreement is maximized by the Borda rule and is minimized ${ }^{6}$ by the Plurality rule and the Antiplurality rule.

Only one reference dealt with the relationship between the four-candidate set and the threecandidate subsets: Gehrlein and Fishburn (1980). First, they established that, the probability of agreement between pairwise comparisons and the collective ranking is maximized by the Borda rule. Next, they computed the mean limit ${ }^{7}$ probability that the collective ranking on three candidates agree with the collective ranking on four candidates. One drawback of their approach is that, they evaluated the likelihood of the same ranking on $\{a, b, c, d\}$ and $\{a, b, c\}$ regardless of the position of $d$ in the four-candidate ranking; they only cared about the possibility of lifting up the initial ranking on $\{a, b, c\}$ to the superset.

Our objective in this paper will be to derive the probability of any ranking on the threecandidate subset given that any candidate has been removed from the four-candidate $\operatorname{set}^{8}$. In this paper, we enrich Gehrlein and Fishburn (1980)'s analysis 1) by obtaining exact probabilities of consistency depending on the original position of the removed candidate; 2) by deriving in each case, the likelihood of all the possible rankings on subsets. Our probability computations not only lead us to make an hierarchy of the main scoring rules according to their stability; we also derive from them, the likelihood of the two electoral paradoxes for committee elections presented above.

\footnotetext{
${ }^{6}$ Gehrlein and Fishburn $(1980,1981)$ showed that with three candidates $a, b, c$, given that the collective ranking is $a b c$, the limit probability to have $a$ majority preferred to $b$ (or $b$ majority preferred to $c$ ) is $85.3 \%$ for the Borda rule and $75.5 \%$ for the Plurality rule and the Antiplurality rule; the limit probability to have $a$ majority preferred to $c$ is $96.9 \%$ for the Borda rule and $90.1 \%$ for the Plurality rule and the Antiplurality rule.

${ }^{7}$ When the electorate tends to infinity.

${ }^{8}$ Notice that, in Gehrlein and Fishburn $(1980,1981)$, Fishburn $(1981)$ and Saari $(1987,1988,1990,1996)$ as it will be the case in this paper, when a candidate is removed, it will not be for strategic or for manipulation purpose as in Tideman (1987) or Dutta et al. (2001). Removing a candidate is strategic if the objective is to improve the ranking of a certain candidate. We will also show that, our results hold for situations in which a new candidate is added in a three-candidate elections. Results on related issue have been recently discovered by Chevaleyre et al. (2012), Lang et al. (2013). We are not going to say more about this point since we are not concerned with the strategic aspect of the withdrawing of one or more candidates.
} 
The rest of the paper is structured as follows : Section 2 is devoted to basic notations and definitions. In Section 3, we motivate the paper by considering some examples showing how the collective rankings over proper subsets of a set of alternatives can be consistent or not with the collective ranking of this set. In Section 4, we evaluate this event in the four-alternatives case using the impartial culture condition. In Section 5, we provide the formal definitions of the PSP and the LMP and we then derive their likelihood in four-candidate elections and two-member committees as a consequence of our probability computations. Section 6 concludes. Details on probability computations are provided in Appendix.

\section{Notation and definitions}

\subsection{Preferences}

Let $N$ be the set of $n$ voters $(n \geq 2)$ and $A$ the set of $m$ alternatives or candidates, $m \geq 3$. The binary relation $R$ over $A$ is a subset of the cartesian product $A \times A$. For $a, b \in A$, if $(a, b) \in R$, we write $a R b$ to say that " $a$ is at least good as $b$ ". $\neg a R b$ is the negation of $a R b$. If we have $a R b$ and $\neg b R a$, we will say that " $a$ is better or strictly preferred to $b$ ". In this case, we write $a P b$ with $P$ the asymmetric component of $R$. The preference profile $\pi=\left(P_{1}, P_{2}, \ldots, P_{i}, \ldots, P_{n}\right)$ gives all the linear orders ${ }^{9}$ of all the $n$ voters on $A$ where $P_{i}$ is the strict ranking of a given voter $i$ over $A$. When we consider the preference of voter $i$ on $B \subset A$, we will simply use the restriction of $P_{i}$ to $B$. The set of all the preference profiles of size $n$ on $A$ is denoted by $\mathcal{P}(A)^{n}$. A voting situation $\tilde{n}=\left(n_{1}, n_{2}, \ldots, n_{t}, \ldots, n_{m !}\right)$ indicates the number of voters for each linear order such that $\sum_{t=1}^{m !} n_{t}=n$. In the subsequent, we simply write $\left(\begin{array}{c}a \\ b \\ c \\ d\end{array}\right)$ or $a b c d$ to say that $a$ is strictly preferred to $b, b$ strictly preferred to $c$ and $c$ strictly preferred to $d$. Table 1 gives the labels of all the 24 types of linear orders with four candidates.

Table 1: Labels of preferences on $A=\{a, b, c, d\}$

\begin{tabular}{|cccccccccccc|}
\hline$n_{1}$ & $n_{2}$ & $n_{3}$ & $n_{4}$ & $n_{5}$ & $n_{6}$ & $n_{7}$ & $n_{8}$ & $n_{9}$ & $n_{10}$ & $n_{11}$ & $n_{12}$ \\
\hline$a$ & $a$ & $a$ & $a$ & $a$ & $a$ & $b$ & $b$ & $b$ & $b$ & $b$ & $b$ \\
$b$ & $b$ & $c$ & $c$ & $d$ & $d$ & $a$ & $a$ & $c$ & $c$ & $d$ & $d$ \\
$c$ & $d$ & $b$ & $d$ & $b$ & $c$ & $c$ & $d$ & $a$ & $d$ & $a$ & $c$ \\
$d$ & $c$ & $d$ & $b$ & $c$ & $b$ & $d$ & $c$ & $d$ & $a$ & $c$ & $a$ \\
\hline \hline$n_{13}$ & $n_{14}$ & $n_{15}$ & $n_{16}$ & $n_{17}$ & $n_{18}$ & $n_{19}$ & $n_{20}$ & $n_{21}$ & $n_{22}$ & $n_{23}$ & $n_{24}$ \\
\hline$c$ & $c$ & $c$ & $c$ & $c$ & $c$ & $d$ & $d$ & $d$ & $d$ & $d$ & $d$ \\
$a$ & $a$ & $b$ & $b$ & $d$ & $d$ & $a$ & $a$ & $b$ & $b$ & $c$ & $c$ \\
$b$ & $d$ & $a$ & $d$ & $a$ & $b$ & $b$ & $c$ & $a$ & $c$ & $a$ & $b$ \\
$d$ & $b$ & $d$ & $a$ & $b$ & $a$ & $c$ & $b$ & $c$ & $a$ & $b$ & $a$ \\
\hline
\end{tabular}

When the number of voters who rank $a$ before $b$ is greater than that of those who rank $b$ before $a, a$ is said to be majority preferred to $b$ for the profile $\pi$; we denote it by $a M(\pi) b$ or simply $a M b$.

\footnotetext{
${ }^{9} \mathrm{~A}$ linear order is a binary relation that is transitive, complete and antisymmetric. The binary relation $R$ on $A$ is transitive if for $a, b, c \in A$, if $a R b$ and $b R c$ then $a R c$. $R$ is antisymmetric if for all for $a \neq b, a R b \Rightarrow \neg b R a$; if we have $a R b$ and $b R a$, then $a=b . R$ is complete if and only if for all $a, b \in A$, we have $a R b$ or $b R a$.
} 


\subsection{Scoring rules}

A scoring rule is a voting system that gives points to candidates in accordance with the position they occupy in voters' rankings. We denote by $w=\left(w_{1}, w_{2}, w_{3}, \ldots, w_{j}, \ldots, w_{m}\right)$ the normalized scoring vector associated to the voting system such that $w_{1}=1 \geq w_{2} \geq \ldots \geq w_{j} \geq \ldots \geq w_{m}=0$. The total number of points received by a candidate defines his score for the considered rule. Let $r(i, a, A)$ be the rank of candidate $a \in A$ in voter $i$ 's ranking ${ }^{10}$.

$$
r(i, a, A)=\sharp\left\{z \in A: z P_{i} a\right\}+1
$$

Given the voting system $w$, for a candidate $a \in A$, his total score is given by :

$$
S(A, w, \pi, a)=\sum_{i=1}^{n} w_{r(i, a, A)}
$$

We denote by $R(A, w, \pi)(R(A, \pi)$ for short) the collective ranking on $A$ given the profile $\pi$ for the scoring vector $w$. In $R(A, \pi)$, the candidate with the greatest total score appears at the top and the one with lowest total score is ranked last.

The most famous scoring rules are:

- The vector $w$ such that $w_{2}=\ldots=w_{m}=0$ defines the simple Plurality rule: voters only cast a vote for their top ranked candidate. For $m=4$, the scoring vector of the simple Plurality rule is $w_{P}=(1,0,0,0)$.

- For $w_{2}=\ldots=w_{m-1}=1$, we obtain the Antiplurality rule under which each voter vote for all the candidates except his bottom ranked candidate; with $m=4$, the scoring vector of the Antiplurality rule is $w_{A p}=(1,1,1,0)$.

- With $m$ candidates, the Borda rule gives $\frac{m-j}{m-1}$ points to a candidate each time he is ranked $j$-th; then, the associated scoring vector is $w=\left(1, \frac{m-2}{m-1}, \ldots, \frac{m-j}{m-1}, \ldots, \frac{1}{m-1}, 0\right)$. In the fourcandidate case, the tally of the Borda rule is $w_{B o r}=\left(1, \frac{2}{3}, \frac{1}{3}, 0\right)$.

- Under the Limited Voting, voters vote for exactly $g$ candidates $(0<g<m)^{11}$. For $g=1$, the Limited Voting is equivalent to the Simple Plurality rule and to the Antiplurality for $g=m-1$. In this paper, as $m=4$, we will take $g=2$. So, the associated tally is $w_{L V}=(1,1,0,0)$.

For all $B \subseteq A$ with $|B| \geq 2$, we define $w^{B}$ the scoring vector of dimension $|B|$ we use to rank alternatives of the subset $B$. As with $w$, we have $w^{B}=\left(w_{1}^{B}, w_{2}^{B}, \ldots, w_{\sharp B}^{B}\right)$ such that $w_{1}^{B}=1 \geq$ $w_{2}^{B} \geq \ldots \geq w_{\sharp B}^{B}=0$. So, for a given subset $B$, the preference $P_{i}$ of voter $i$ is now defined by its restriction to $B$. Hence, given $w^{B}$, for a candidate $a \in B$, his score is given by :

$$
S(B, w, \pi, a)=\sum_{i=1}^{n} w_{r(i, a, B)}^{B}
$$

We denote by $R(\pi, B)$ the collective ranking on $B$ when the profile $\pi$ is restricted to $B$.

\footnotetext{
${ }^{10}$ In a similar way we defines $r(i, a, B)$ for $a \in B \subseteq A$.

${ }^{11}$ For an overview on this voting system and those related to it, see Dummet (1984). These rules are also called $g$-approval rules
} 
In this paper, we will focus on $|A|=4$ and $|B|=3$. So, if $A=\{a, b, c, d\}$ then $B \in$ $\{\{a, b, c\},\{a, b, d\},\{a, c, d\},\{b, c, d\}\}$. Assuming that the same scoring rule is used on both the whole set and on the three candidate subsets, Table 2 gives for each of the famous voting rules stated above, the associated tallies.

Table 2: Voting rules and tallies

\begin{tabular}{||c||c||c||}
\hline \multicolumn{1}{||c||}{} & \multicolumn{2}{c|}{ Tallies } \\
\hline Voting rules & $w$ & $w^{B}$ \\
\hline \hline Plurality & $(1,0,0,0)$ & $(1,0,0)$ \\
\hline \hline Antiplurality & $(1,1,1,0)$ & $(1,1,0)$ \\
\hline \hline Borda & $\left(1, \frac{2}{3}, \frac{1}{3}, 0\right)$ & $\left(1, \frac{1}{2}, 0\right)$ \\
\hline \hline Limited Voting & $(1,1,0,0)$ & $(1,1,0)$ \\
\hline \hline
\end{tabular}

In the sequel, our analysis, will focus on the whole family of scoring rules. So, as we are going to analyze four-candidate elections, we will use $w=\left(1, w_{2}, w_{3}, 0\right)$ and $w^{B}=\left(1, w_{2}^{B}, 0\right)$.

\section{Consistency and inconsistency}

Let $R(\pi, A / B)$ be the restriction of the collective ranking over $A$ on candidates in $B$. When restricting the profile $\pi$ from $A$ to $B$ the following events can occur :

- $R(\pi, B)=R(\pi, A / B)$. The candidates in the collective ranking on $B$ appear in the same order as in the collective ranking on $A$. In a such case, we will say that the scoring rule $w^{B}$ agree or is consistent with the scoring rule $w$.

- $R(\pi, B) \neq R(\pi, A / B)$. The candidates in the collective ranking on $B$ do not appear in the same order as in the collective ranking on $A$. In a such case, we will say that the scoring rule $w^{B}$ is not consistent with the scoring rule $w$. Even worse, the candidates in the collective ranking on $B$ may appear in the reversed order of that of the collective ranking on $A$, as shown in Example 1 .

Example 1. For $A=\{a, b, c, d\}$, consider the following preference profile with 102 voters $^{12}$.

\begin{tabular}{|ccccccccccccc|}
\hline \hline 12 & 6 & 6 & 3 & 6 & 12 & 6 & 7 & 6 & 12 & 14 & 6 & 6 \\
\hline \hline$a$ & $a$ & $a$ & $a$ & $b$ & $b$ & $b$ & $c$ & $c$ & $c$ & $d$ & $d$ & $d$ \\
$b$ & $b$ & $d$ & $d$ & $c$ & $c$ & $d$ & $a$ & $a$ & $d$ & $a$ & $b$ & $c$ \\
$c$ & $d$ & $b$ & $c$ & $a$ & $d$ & $c$ & $b$ & $d$ & $a$ & $b$ & $c$ & $a$ \\
$d$ & $c$ & $c$ & $b$ & $d$ & $a$ & $a$ & $d$ & $b$ & $b$ & $c$ & $a$ & $b$ \\
\hline \hline
\end{tabular}

For a given scoring vector $w=\left(1, w_{2}, w_{3}, 0\right)$, we get,

$$
S(A, w, \pi, a)=27+27 w_{2}+24 w_{3}, \quad S(A, w, \pi, b)=24+24 w_{2}+27 w_{3}
$$

${ }^{12}$ Notice that similar example with fewer number of voters may exist. 


$$
S(A, w, \pi, c)=25+24 w_{2}+27 w_{3}, \quad S(A, w, \pi, d)=26+27 w_{2}+24 w_{3}
$$

Since $w_{2} \geq w_{3}$, it can be easily checked that $R(\pi, A)=a d c b$.

Suppose that $b$ is dropped. Using the scoring vector $w^{B}=\left(1, w_{2}^{B}, 0\right)$ on $B=\{a, c, d\}$, we have

$$
S\left(B, w^{B}, \pi, a\right)=27+33 w_{2}^{B}, \quad S\left(B, w^{B}, \pi, b\right)=43+30 w_{2}^{B}, \quad S\left(B, w^{B}, \pi, c\right)=33+39 w_{2}^{B}
$$

Since $R(\pi, B)=c d a$ for $w^{B}=0$ and $w^{B}=1$, it comes that $R(\pi, B)=c d a$ for all $w^{B}$.

So, with this preference profile, for every couple $\left(w, w^{B}\right)$ of scoring vectors, given that $R(\pi, A)=a d c b$, removing $b$ leads to a complete reversal ranking on the remaining candidates.

Assume that, we want to elect a committee of two members out of $A=\{a, b, c, d\}$. Given $R(\pi, A)=a d c b$, the appointed two-member committee is $\{a, d\}$. Let us assume that candidate $a$ who is elected decided to resign and that a new ballot is taken ceteris paribus on $A=\{b, c, d\}$. Using the scoring vector $w^{B}=\left(1, w_{2}^{B}, 0\right)$ on $B=\{b, c, d\}$, we end with

$$
S\left(B, w^{B}, \pi, b\right)=42+33 w_{2}^{B}, \quad S\left(B, w^{B}, \pi, c\right)=25+39 w_{2}^{B}, \quad S\left(B, w^{B}, \pi, d\right)=35+30 w_{2}^{B}
$$

It comes that $R(\pi, B)=b d c$ for all $w^{B}$, then new appointed committee is $\{b, d\}$. Candidate $c$ who was ranked third in the previous ballot (he is called the Prior Successor) is not appointed : this defines what we called the Prior Successor Paradox. The reader can check that with our preference profile, there is no possibility for the Leaving Member Paradox. An example illustrating the Leaving Member Paradox is provided by Staring (1986) for a voting system under which voters vote for as may candidates as there are vacancies to be filled (i.e the Limited Voting $)^{13}$.

All in all, what kind of inconsistencies can appear for a given generalized scoring rule $\omega^{14}$ ? Saari studied extensively this issue in a series of seminal papers (Saari, 1987, 1988, 1990, 1996). He showed that if an inconsistency occurs for some profile with the Borda count, it will also occur (for the same profile or a different one) for any other generalized scoring rule. Moreover, the Borda count uniquely minimizes the number of possible words in this class of voting rules ${ }^{15}$. Though the Borda count can be viewed as the optimal scoring rule, it is not free from severe paradoxes such as the reversal paradox displayed in Example 1. What kind of relationships can we expect when we use the Borda count? The answer is the generalization of a well known result in social choice literature : The Condorcet winner (i.e the candidate who is able to defeat any other opponent in pairwise comparison) is never ranked last by the Borda count and the Condorcet loser (i.e the candidate who is defeated by any other alternative) is never ranked first by the Borda count ${ }^{16}$. Saari $(1987,1990,1996)$ 's result try to establish the superiority of the Borda count in the class of scoring rule when one wishes to minimizes the types of inconsistencies we can observe across subsets. However, they do not tell us whether the likelihood of paradoxes are rare oddities or not, and whether the Borda count also minimizes the probability of the different paradoxes. The vast literature on the relationships between pairwise majority and scoring rules gives a positive answer to this last issue (See Gehrlein and Lepelley (2010) for a survey). For

\footnotetext{
${ }^{13}$ Preference profiles with four candidates exhibiting the Leaving Member Paradox can be readily constructed for any $w=\left(1, w_{2}, w_{3}, 0\right)$ and $w^{B}=\left(1, w_{2}^{B}, 0\right)$.

${ }^{14}$ A generalized scoring rule $\omega=\left(w^{B^{1}}, w^{B^{2}}, \ldots, w^{B^{j}}, \ldots, w^{B^{2^{m}-(m+1)}}\right)$ is the collection of scoring vectors used for each proper subset $B$ of $A$.

${ }^{15}$ However, this is not the case if we seek for generalized voting rules in other families.

${ }^{16}$ An early version of this result can be tracked back to Nanson (1882). Modern proofs are proposed by Smith (1973) and Fishburn and Gehrlein (1976).
} 
almost all the a priori probability distribution one can imagine on the set of preferences profiles ${ }^{17}$ the Borda count is the most likely to lift up the results on pairwise comparisons to supersets ${ }^{18}$.

By estimating the likelihood of consistent and inconsistent rankings on four candidate and three candidate subsets, the current paper wishes to examine whether the preeminence of the Borda count will stand again. The analysis of consistent and inconsistent rankings will allow us examine the Prior Successor Paradox and the Leaving Member Paradox (in Section 5). Then, from our results we will also check the preeminence of the Borda count. First of all, we need to set an a priori assumption on the likelihood of the different profiles. In a first step, we chose the most common one, namely, the Impartial Culture assumption.

\section{Probabilities of consistency with four alternatives}

\subsection{A probabilistic model: The impartial culture}

One of the most used assumptions in the social choice literature when computing the likelihood of given events is the Impartial Culture (IC). Under IC, it is assumed that, each voter chooses her preference according to a uniform probability distribution. It gives a probability $\frac{1}{m !}$ for each ranking to be chosen independently. The likelihood of a given voting situation $\tilde{n}=\left(n_{1}, n_{2}, \ldots, n_{t}, \ldots, n_{m !}\right)$ is

$$
\operatorname{Prob}\left(\tilde{n}=\left(n_{1}, n_{2}, \ldots, n_{t}, \ldots, n_{m !}\right)\right)=\frac{n !}{\prod_{i=1}^{m !} n_{i} !} \times(m !)^{-n}
$$

For more details about the IC and other probabilistic assumptions, see among others Gehrlein and Fishburn (1976), Berg and Lepelley (1994), Gehrlein and Lepelley (2010).

\subsection{The consistency probabilities : Gehrlein and Fishburn's results}

Using the IC assumption, Gehrlein and Fishburn (1980) have computed in four-candidate elections 1 )-the limit probability that the pairwise comparisons are consistent with the collective ranking and 2)-the mean limit probability that the collective ranking on three candidates agrees with the collective ranking on four candidates.

Consider $A=\{a, b, c, d\}$ a set of four alternatives and $B$ a proper subset of $A$ with two alternatives such that $B \in\{\{a, b\},\{a, c\},\{a, d\},\{b, c\},\{b, d\},\{c, d\}\}$. Let us denote by $q\left(\left(w, w^{B}\right), x y\right)$ $(x, y \in A, x \neq y)$ the limit probability under IC that the pairwise comparison between $x$ and $y$ is consistent with the way $x$ and $y$ appear in the collective ranking on $A$. So, if $a b c d$ is the collective ranking, $q\left(\left(w, w^{B}\right), a b\right)$ will be the probability that $a$ is majority preferred to $b$ or the probability that $a$ still ranked before $b$ when the two other candidates are removed. Gehrlein and Fishburn (1980) provided the expression of each of the $q\left(\left(w, w^{B}\right), x y\right)$. We are not going to recall these expressions here. They showed that

$$
q\left(\left(w, w^{B}\right), a b\right)=q\left(\left(w, w^{B}\right), c d\right) \text { and } q\left(\left(w, w^{B}\right), a c\right)=q\left(\left(w, w^{B}\right), b d\right)
$$

\footnotetext{
${ }^{17}$ We only consider the probability models that are neutral, i.e, that treat equally all alternatives and all the rankings.

${ }^{18} \mathrm{~A}$ few exception has been reported concerning the selection of the Condorcet winner in the three candidate elections. Under an assumption called the Impartial Anonymous Culture, Cervone et al. (2005) showed that though the Borda coun is close to be optimal, the real optimum is obtained with $v=(1,0.37225,0)$ as $n$ goes to infinity. The optimality of the Borda count can also be contested by Approval Voting in some specific scenarios when one considers indifferent voters, as in Diss et al. (2010).
} 
Also, they showed that each $q\left(\left(w, w^{B}\right), x y\right)$ is maximized by the Borda rule and minimized by the Plurality rule and the Antiplurality rule. In Table 3, we report their results for the Borda rule, the Plurality rule, the Antiplurality rule and for the Limited Voting.

Table 3: Scoring rules and consistency probabilities over two-alternatives subsets when the collective ranking is $a b c d$

\begin{tabular}{||c||c||c||c||c||}
\cline { 2 - 5 } \multicolumn{1}{c|}{} & $q\left(\left(w, w^{B}\right), a b\right)$ & $q\left(\left(w, w^{B}\right), a c\right)$ & $q\left(\left(w, w^{B}\right), a d\right)$ & $q\left(\left(w, w^{B}\right), b c\right)$ \\
\hline \hline Borda & 0.799847 & 0.924842 & 0.980402 & 0.767058 \\
\hline \hline Plurality & 0.680513 & 0.796887 & 0.893273 & 0.651925 \\
\hline Antiplurality & & 0.859501 & 0.943660 & 0.699058 \\
\hline \hline Limited Voting & 0.731399 & 0.1904 \\
\hline \hline
\end{tabular}

It comes from Table 3 that the Limited voting performs better than the Plurality rule and the Antiplurality rule.

In the four-candidate case, Gehrlein and Fishburn (1980) computed $^{19}$ the limit probability given the collective ranking on $A=\{a, b, c, d\}$ that the new collective ranking between $a, b$ and $c$ is consistent with the former one after alternative $d$ is removed no matter his position in that ranking ${ }^{20}$. They showed that this probability is maximized by the Borda rule and minimized by the two Plurality-Antiplurality score vectors combination i.e $w=(1,0,0,0)$ and $w^{B}=(1,1,0)$. In the four-candidate case, Gehrlein and Fishburn (1980)'s results are somewhat limited in scope since they leave some questions unanswered. Is the agreement probability the same when the removed candidate was first, second, third or last in the original ranking? When a four-alternative set is restricted to a three-alternative proper subset, what is the likelihood of different rankings to occur? The answers follow.

\subsection{Consistency probabilities : generalized results for subsets of three alternatives}

This section completes Gehrlein and Fishburn (1980)'s results by computing in four-candidate elections, the limit probability (under IC) of a given ranking to occur after one candidate is removed depending on the position of the removed candidate in the original collective ranking.

With $A=\{a, b, c, d\}$ we will consider the following collective rankings : $a b c d, a b d c, a d b c$ and $d a b c$. Then, we suppose that $d$ is removed and we compute the likelihood of each of the following rankings to occur : $R_{1}: a b c, R_{2}: a c b, R_{3}: b a c, R_{4}: b c a, R_{5}: c a b, R_{6}: c b a$. Our analysis takes into account all the pairs $\left(w, w^{B}\right)$ of scoring voting vectors then we will provide the results for the scoring rules presented in Table 2 .

With $A=\{a, b, c, d\}$, we denote by $P_{\infty}^{I C}\left(\left(w, w^{B}\right), a b c / a b c d\right)$ the limit probability under IC, that the ranking $a b c$ occurs when $d$ the last ranked candidate of the collective ranking on $A=$ $\{a, b, c, d\}$ is removed given the couple of tallies $\left(w, w^{B}\right)$. In the same way we define $P_{\infty}^{I C}\left(\left(w, w^{B}\right), a b c / a b d c\right)$, $P_{\infty}^{I C}\left(\left(w, w^{B}\right), a b c / a d b c\right), P_{\infty}^{I C}\left(\left(w, w^{B}\right), a b c / d a b c\right)$, and so on. One can notice that, $P_{\infty}^{I C}\left(\left(w, w^{B}\right), a b c / a b c d\right)$ is the probability that the three-candidate's collective ranking is consistent with the four-candidate's collective ranking when $d$ is removed; while $P_{\infty}^{I C}\left(\left(w, w^{B}\right), c b a / a b c d\right)$ is the probability that the three-candidate's collective ranking is totaly the reverse of that with four candidates after $d$ is removed (as shown in Example 1).

\footnotetext{
${ }^{19}$ They provided the expression of the limit probability that we are not going to report here due to space constraints.

${ }^{20}$ This means that the collective ranking on $A=\{a, b, c, d\}$ could be $a b c d$ or $a b d c$ or $a d b c$ or $d a b c$.
} 
Due to space constraints, were are not going to report the expressions of the probability formulas and their proofs here. All these details are provided in a supplement note (see Kamwa and Merlin (2015) $)^{21}$. In this note, we also provide a MAPLE-sheet of our computation program. We obtained general formulas for each of the event analyzed here. In the supplement note, we only provide the general formula for $P_{\infty}^{I C}\left(\left(w, w^{B}\right), a b c / a b c d\right)$. We need to raise some important remarks that follow from our computations : for $A=\{a, b, c, d\}$ and for all pairs $\left(w, w^{B}\right)$ of scoring rules, we found that

$$
\begin{aligned}
& P_{\infty}^{I C}\left(\left(w, w^{B}\right), a b c / a b c d\right)=P_{\infty}^{I C}\left(\left(w, w^{B}\right), a b c / d a b c\right) \\
& P_{\infty}^{I C}\left(\left(w, w^{B}\right), a b c / a b d c\right)=P_{\infty}^{I C}\left(\left(w, w^{B}\right), a b c / a d b c\right) \\
& P_{\infty}^{I C}\left(\left(w, w^{B}\right), c b a / a b c d\right)=P_{\infty}^{I C}\left(\left(w, w^{B}\right), c b a / d a b c\right) \\
& P_{\infty}^{I C}\left(\left(w, w^{B}\right), c b a / a b d c\right)=P_{\infty}^{I C}\left(\left(w, w^{B}\right), c b a / a d b c\right)
\end{aligned}
$$

and for $(\phi, \tau)=(a c b, b a c)$ or $(c a b, b c a)$,

$$
\begin{aligned}
& P_{\infty}^{I C}\left(\left(w, w^{B}\right), \phi / a b c d\right)=P_{\infty}^{I C}\left(\left(w, w^{B}\right), \tau / d a b c\right) \\
& P_{\infty}^{I C}\left(\left(w, w^{B}\right), \phi / a b d c\right)=P_{\infty}^{I C}\left(\left(w, w^{B}\right), \tau / a d b c\right) \\
& P_{\infty}^{I C}\left(\left(w, w^{B}\right), \phi / a d b c\right)=P_{\infty}^{I C}\left(\left(w, w^{B}\right), \tau / a b d c\right) \\
& P_{\infty}^{I C}\left(\left(w, w^{B}\right), \phi / d a b c\right)=P_{\infty}^{I C}\left(\left(w, w^{B}\right), \tau / a b c d\right)
\end{aligned}
$$

For each of our general formulas, we tried to find the pair $\left(w, w^{B}\right)$ of scoring vectors at which it is maximized or minimized. The conclusion was quite the same. The following theorem tells us more.

Theorem 1. Given the pairs $\left(w, w^{B}\right)$ of scoring vectors, the collective ranking on $A=\{a, b, c, d\}$ and the position of the removed candidate, the probability of consistency is maximized by the the Borda rule $\left(w=\left(1, \frac{2}{3}, \frac{2}{3}, 0\right), w^{B}=\left(1, \frac{1}{2}, 0\right)\right)$ and minimized by the two Plurality-Antiplurality score vectors combination $\left(w=(1,0,0,0), w^{B}=(1,1,0)\right)$ and $\left(w=(1,1,1,0), w^{B}=(1,0,0)\right)$. The probability of inconsistency is minimized by the the Borda rule and maximized by the two Plurality-Antiplurality score vectors combinations.

What we learn from Theorem 1 is that, it is more difficult under the Borda rule than under any other scoring rules s to end with a total reversal ranking (or with a ranking swapping candidates) on the three-candidate set given a collective ranking on a four-candidate set, when one candidate is removed.

In Tables 4 to 6 , we report the consistency probabilities values for each the scoring rules presented in Table 2.

Given the collective ranking on $a, b, c, d$, let us denote by $P_{\infty}^{I C}\left(\left(w, w^{B}\right), a, b, c, d / R_{i}\right)$ the limit probability to end with $R_{i}(i=1 \ldots 6)$ as the new collective ranking on $a, b, c$, independently of the

\footnotetext{
${ }^{21}$ This supplement note was made under the suggestion of one anonymous reviewer who suggested to reduce the length of the paper. We thank him for this suggestion.
} 
Table 4: Consistency probabilities over subsets for the Borda rule on $A=\{a, b, c, d\}$

\begin{tabular}{|c|c|c|c|c|c|c|}
\hline collective rankings & $a b c$ & $a c b$ & $b a c$ & $b c a$ & $c a b$ & $c b a$ \\
\hline$a b c d$ & 0.719518 & 0.138512 & 0.111617 & 0.011626 & 0.011888 & 0.006836 \\
\hline$a b d c$ & 0.840425 & 0.028084 & 0.127008 & 0.002214 & 0.001408 & 0.000858 \\
\hline$a d b c$ & 0.840425 & 0.127008 & 0.028084 & 0.001408 & 0.002214 & 0.000858 \\
\hline$d a b c$ & 0.719518 & 0.111617 & 0.138512 & 0.011888 & 0.011626 & 0.006836 \\
\hline$P\left(R_{i}\right)$ & 0.779971 & 0.101305 & 0.101305 & 0.006784 & 0.006784 & 0.003847 \\
\hline
\end{tabular}

Table 5: Consistency probabilities over subsets for the Plurality rule and the Antiplurality rule on $A=\{a, b, c, d\}$

\begin{tabular}{|c||c||c||c||c||c||c||}
\cline { 2 - 7 } collective rankings & $a b c$ & $a c b$ & $b a c$ & $b c a$ & $c a b$ & $c b a$ \\
\hline \hline$a b c d$ & 0.516969 & 0.203037 & 0.171531 & 0.040395 & 0.042127 & 0.025939 \\
\hline$a b d c$ & 0.661087 & 0.089474 & 0.213197 & 0.017253 & 0.011572 & 0.007415 \\
\hline$a d b c$ & 0.661087 & 0.213197 & 0.089474 & 0.011572 & 0.017253 & 0.007415 \\
\hline$d a b c$ & 0.516969 & 0.171531 & 0.203037 & 0.042127 & 0.040395 & 0.025939 \\
\hline$P\left(R_{i}\right)$ & 0.589028 & 0.169309 & 0.169309 & 0.027836 & 0.027836 & 0.016677 \\
\hline \hline \hline
\end{tabular}

position of $d$ the removed candidate. From our computations, we found that

$$
P_{\infty}^{I C}\left(\left(w, w^{B}\right), a, b, c, d / R_{i}\right)=\frac{\begin{array}{c}
P_{\infty}^{I C}\left(\left(w, w^{B}\right), R_{i} / a b c d\right)+P_{\infty}^{I C}\left(\left(w, w^{B}\right), R_{i} / a b c d\right) \\
+P_{\infty}^{I C}\left(\left(w, w^{B}\right), R_{i} / a b c d\right)+P_{\infty}^{I C}\left(\left(w, w^{B}\right), R_{i} / a b c d\right)
\end{array}}{4}
$$

We report the values $P\left(R_{i}\right)$ of the probability $P_{\infty}^{I C}\left(\left(w, w^{B}\right), a, b, c, d / R_{i}\right)$ in the last rows of Tables 4 to 6 . The probability computed by Gehrlein and Fishburn $(1980), P_{\infty}^{I C}\left(\left(w, w^{B}\right), a, b, c, d / R_{1}\right)$ the consistency probability independently of the position of the removed candidate, is somehow a mean value of our consistency probabilities. It follows from Theorem 1 that this probability is maximized by the Borda rule and minimized by the two Plurality rule-Antiplurality rule score vector combinations. This removes the conjecture stated by (Gehrlein and Fishburn, 1980, p.253). Thus, our results complete those of Gehrlein and Fishburn (1980) and offer a broader outlook.

If we compare the values of Table 5 to those of Table 6, it comes that, the Plurality rule and the Antiplurality rule are more consistent and less inconsistent than the Limited Voting. We also

Table 6: Consistency probabilities over subsets for the Limited Voting rule on $A=\{a, b, c, d\}$

\begin{tabular}{|c||c||c||c||c||c||c||}
\cline { 2 - 7 } collective rankings & $a b c$ & $a c b$ & $b a c$ & $b c a$ & $c a b$ & $c b a$ \\
\hline \hline$a b c d$ & 0.412089 & 0.217588 & 0.190647 & 0.065521 & 0.068797 & 0.045355 \\
\hline$a b d c$ & 0.537710 & 0.132448 & 0.243258 & 0.039356 & 0.027916 & 0.018909 \\
\hline$a d b c$ & 0.537710 & 0.243258 & 0.132448 & 0.027916 & 0.039356 & 0.018909 \\
\hline$d a b c$ & 0.412089 & 0.190647 & 0.217588 & 0.068797 & 0.065521 & 0.045355 \\
\hline \hline$P\left(R_{i}\right)$ & 0.474900 & 0.195985 & 0.195985 & 0.050397 & 0.050397 & 0.032132 \\
\hline \hline
\end{tabular}


Table 7: Limit probability for $a$ to remain first

\begin{tabular}{c|c||c||c||}
\cline { 2 - 4 } \multicolumn{1}{c|}{ collective rankings } & Borda & $\begin{array}{c}\text { Plurality } \\
\text { Antiplurality }\end{array}$ & Limited Voting \\
\hline \hline$a b c d$ & 0.858030 & 0.720006 & 0.629677 \\
\hline \hline$a b d c$ & 0.868509 & 0.750561 & 0.670158 \\
\hline \hline$a d b c$ & 0.967433 & 0.874284 & 0.780968 \\
\hline \hline$P_{\text {first }}$ & 0.897990 & 0.781617 & 0.693601 \\
\hline
\end{tabular}

Table 8: Limit probability for $a$ to become last

\begin{tabular}{c|c||c||c||}
\cline { 2 - 4 } \multicolumn{1}{c|}{ collective rankings } & Borda & $\begin{array}{c}\text { Plurality } \\
\text { Antiplurality }\end{array}$ & Limited Voting \\
\hline \hline$a b c d$ & 0.018462 & 0.066334 & 0.110876 \\
\hline \hline$a b d c$ & 0.003072 & 0.024668 & 0.052650 \\
\hline \hline$a d b c$ & 0.002266 & 0.018987 & 0.046825 \\
\hline \hline$P_{\text {last }}$ & 0.007933 & 0.036663 & 0.070117 \\
\hline \hline
\end{tabular}

learn from our general results that, given a collective ranking, when a candidate is removed, it becomes difficult to end with a new collective ranking in which two candidates are swapped as the gap between them increases comparatively to the original ranking. From Tables 4 to 6 , we deduce for each of our scoring rules, $P_{\text {first }}$ and $P_{\text {last }}$ respectively the propensities for a top ranked candidate to remain first (see Table 7) or to become last (see Table 8) after a given candidate is removed.

According to Tables 7 and 8, for each of our scoring rules, when a candidate (except the top ranked one) is removed, it is likely for a top ranked candidate to remain first (on average, $89.79 \%$ for the Borda rule, $78.16 \%$ for the Plurality rule and the Plurality and $69.36 \%$ for the Limited Voting) while it is very difficult for this candidate to become last with the Borda rule (on average $3.67 \%$ for the Plurality rule and the Plurality and $7.01 \%$ for the Limited Voting). Also, for each of our voting rules, as candidates $a$ and $d$ become closer, it is more likely (resp. difficult) for $a$ to remain first (resp. to become last) after $d$ is removed.

We have learnt from Table 3 that the pairwise comparisons are more consistent with the collective ranking under the Limited Voting than under the Plurality and the Antiplurality. When considering the proper subsets of three candidates, we obtain the opposite result. So, we can expect that, with a given set of candidates, when studying the consistency between the collective ranking on this set and what we have on its proper subsets, the hierarchy among the scoring rules may not be the same as the size of the subsets vary.

\section{Evaluating the likelihood of two paradoxes of committees elections}

As already said, our probabilities complete Gehrlein and Fishburn (1980)'s results and give a complete picture on the consistency of collective rankings and those on subsets of alternatives. In this section, we will exploit the exhaustibility of our results of the previous sections. More 
particularly, we will focus on the paradoxes that can occur when one wishes to select a committee of two out of four candidates.

\subsection{Electing committees}

Electoral laws and constitutions of many democratic organizations and countries stipulate that, when electing a committee or a group of representatives (a parliament, a board, etc.), every elected candidate must have at least one substitute. A substitute is supposed to take the place of the elected candidate if for various reasons, he comes to leave. In contrast, there are some organizations that elect boards without provision of substitutes. For some of them, nothing is said on what will be done if one elected member decides to leave. For others, a recourse to new (partial) elections is required in such a case.

Suppose that a ballot with $m$ candidates (at least three) leads to a committee made of the $g$ candidates with the greatest scores ( $g$ at least equal to two) and that there is no provision of substitutes for any elected member of the committee. In such a case, if an elected candidate decides to leave, there are three possible ways to fill the empty chair ceteris paribus ${ }^{22}$ :

- Rule $1\left(R_{1}\right)$ simply nominate the candidate ranked $g+1$-th in the original ballot;

- Rule $2\left(R_{2}\right)$ take a new ballot on the remaining $m-1$ candidates and pick the $g$ greatest scores;

- Rule $3\left(R_{3}\right)$ take a partial ballot on the others $m-g$ non elected candidates and take the one who scores the best.

It is clear that rule $R_{1}$ is the natural way to replace the leaving candidate. Nonetheless, the different rules can lead to different committees. How often? In order to answer this issue, we define and analyze the discrepancies between the Rule 1 and the two others possible ways to fill an empty chair.

\subsection{The Prior Successor Paradox}

Suppose that we want to elect a committee of $g$ members $(2 \leq g<m-1)$ and that the elected candidates are those with the $g$ greatest scores. We denote by $C^{g}$ the set of all subsets of $A$ of size $g$; we denote by $C^{*} \in C^{g}$ the elected committee. If a candidate leaves $C^{*}$, we will denote by $\hat{C}^{*} \in C^{g} \backslash C^{*}$ the new elected committee after a ballot has be taken to replace this leaving candidate.

Since we have supposed that the elected committee is formed by the candidates with the $g$ greatest scores (in particular with scoring rules), we define the Prior Successor as the candidate with the $g+1-$ th best score.

Definition 1. (Prior Successor) Let $m \geq 3$ and $1 \leq g \leq m-1$. For $C^{*} \in C^{g}$, a candidate $a$ is called the Prior Successor if $a \notin C^{*}$ and candidate $a$ is the one with the $g+1-$ th greatest score.

The Prior Successor Paradox (PSP) occurs if after a member of the elected committee leaves, a new ballot (given the subset of candidates) leads to a committee containing all the $g-1$ members of the previous committee without the Prior Successor.

\footnotetext{
${ }^{22}$ This means that, voters keep their preferences unchanged on the rest of candidates no matter who leaves.
} 
Table 9: Likelihood of the PSP

\begin{tabular}{|c|c|c|c|}
\hline & \multirow{2}{*}{\multicolumn{2}{|c|}{$\frac{\text { New ballot }}{P S P}$}} & \multirow{3}{*}{$\begin{array}{c}\text { Partial ballot } \\
P_{\mathrm{PSP}}^{I C_{\infty}}\end{array}$} \\
\hline & & & \\
\hline & $P_{\mathrm{PSP}_{1}}^{I C_{\infty}}$ & $P_{\mathrm{PSP}_{2}}^{I C_{\infty}}$ & \\
\hline Borda & 0.123243 & 0.129222 & 0.200153 \\
\hline Plurality & \multirow{2}{*}{0.211926} & \multirow{2}{*}{0.230450} & \multirow{2}{*}{0.319487} \\
\hline Antiplurality & & & \\
\hline Limited Voting & 0.256168 & 0.282614 & 0.268601 \\
\hline
\end{tabular}

Definition 2. (Prior Successor Paradox) Let $m \geq 3,1 \leq g \leq m-1$ and an elected committee $C^{*}$ such that candidate $a$ is the Prior Successor $\left(a \notin C^{*}\right)$. The Prior Successor Paradox occurs if when a candidate $d \in C^{*}$ leaves, we have $a \notin \hat{C}^{*}$ and $C^{*} \backslash\{d\} \subset \hat{C}^{*}$.

Thus, the PSP can occur when one chooses not to just nominate the prior successor as recommended by rule $R_{1}$. If a new ballot is taken (Rule $R_{2}$ ) when an elected candidate leaves, we denote by $P_{\mathrm{PSP}_{j}}^{I C_{\infty}}\left(w, w^{B}\right)$ the limit probability under IC of the PSP given that the leaving candidate is the one who was ranked $j$-th $(j \leq g)$ in the collective ranking. This is also the limit probability that the new elected committee differs from that recommended by rule $R_{1}$. Given that the collective ranking is $d a b c$ and that the two-member committee is $(a, d)$, if candidate $d$ leaves, the $\operatorname{PSP}_{1}\left(w, w^{B}\right)$ will occur after a new ballot if the new elected two-member committee is $(a, c)$ i.e the new collective ranking is $a c b$ or $c a b$. So,

$$
P_{\mathrm{PSP}_{1}}^{I C_{\infty}}\left(w, w^{B}\right)=P_{\infty}^{I C}\left(\left(w, w^{B}\right), a c b / d a b c\right)+P_{\infty}^{I C}\left(\left(w, w^{B}\right), c a b / d a b c\right)
$$

If candidate $a$ leaves, the $\mathrm{PSP}_{2}$ will occur after a new ballot if the new elected two-member committee is $(d, c)$ i.e the new collective ranking is $d c b$ or $c d b$. So,

$$
\begin{aligned}
P_{\mathrm{PSP}_{2}}^{I C_{\infty}}\left(w, w^{B}\right) & =P_{\infty}^{I C}\left(\left(w, w^{B}\right), d c b / d a b c\right)+P_{\infty}^{I C}\left(\left(w, w^{B}\right), c d b / d a b c\right) \\
& =P_{\infty}^{I C}\left(\left(w, w^{B}\right), a c b / a d b c\right)+P_{\infty}^{I C}\left(\left(w, w^{B}\right), c a b / a d b c\right)
\end{aligned}
$$

As the probabilities $P_{\mathrm{PSP}_{j}}^{I C_{\infty}}\left(w, w^{B}\right)$ are sums of probabilities of inconsistency, it follows directly from Theorem 1 that there are minimized by the Borda rule and maximized by the Plurality rule-Antiplurality rule combination. For four-candidate elections and two-member committees, Table 9 gives the values of $P_{\mathrm{PSP}_{1}}^{I C_{\infty}}\left(w, w^{B}\right)$ (and that of $P_{\mathrm{PSP}_{2}}^{I C_{\infty}}\left(w, w^{B}\right)$ ) which is the probability of the PSP when the leaving candidate is the one with the highest (resp. second best) score in the elected two-member committee. According to these probabilities, the Limited voting appears to be more vulnerable to both the $\mathrm{PSP}_{1}$ and the $\mathrm{PSP}_{2}$ (with respectively $25.62 \%$ and $28.26 \%$ ) than the Plurality and the Antiplurality rule (21.19\% and $23.04 \%)$.

Using our general formulas, we come to the conclusion that, in four candidate-election and for two-member committees, the PSP is lightly likely to occur if the candidate with the highest score in the committee leaves. So, the Also, for each of these six scoring rules, the $\mathrm{PSP}_{1}$ is less likely than the $\mathrm{PSP}_{2}$.

Notice that we did not derive the probability of the PSP no matter the ranking of the leaving candidate. Assume for $A=\{a, b, c, d\}$ that the elected committee is $\{a, b\}$; nothing tell us that the PSP only occurs when $a$ leaves and not when $b$ leaves and vice-versa. So, we need more 
information than what are displayed in our tables : the joint probabilities ${ }^{23}$.

What do we have in case of a partial ballot (Rule $R_{3}$ )? With four candidates and two-member committee, given that the collective ranking is $a b c d$ and that the two-member committee is $(a, b)$, the PSP will occur with a partial ballot after a member of the elected committee leaves if and only if candidate $c$ who is the Prior Successor is beaten in pairwise majority by candidate $d$. So, probability $P_{\mathrm{PSP}}^{I C_{\infty}}\left(w, w^{B}\right)$ of the PSP in this case is the probability that the pairwise majority between the two last ranked candidates is not consistent with the collective ranking on the four candidates. It comes that, $P_{\mathrm{PSP}}^{I C_{\infty}}\left(w, w^{B}\right)$ is the complementary of the results obtained by Gehrlein and Fishburn (1980) (see Table 3 ) on the consistency between pairwise majority and the collective ranking in four-candidate elections. So, with $a b c d$ as the collective ranking,

$$
P_{\mathrm{PSP}}^{I C_{\infty}}\left(w, w^{B}\right)=1-q\left(\left(w, w^{B}\right), c d\right)
$$

The last column of Table 9 gives the values of $P_{\mathrm{PSP}}^{I C_{\infty}}\left(w, w^{B}\right)$ for each of our analyzed scoring rules. It is $20.01 \%$ for the Borda rule (20.01\%); according to these values, the Plurality rule and the Antiplurality rule are the more likely with $31.95 \%$ the Limited Voting $(26.86 \%)$ to produce the PSP in case of a partial ballot.

We will now deal with another paradox of committees that is more stronger than the PSP : the Leaving Member Paradox (LMP).

\subsection{The Leaving Member Paradox}

According to Staring (1986), when voters vote for exactly $g$ candidates in order to elect a board of $\operatorname{size}^{24} \mathrm{~g}$, if a new ballot is taken, ceteris paribus, after an elected candidate leaves (this is consistent with rule $R_{2}$ ), we can end up with a committee that does not contain one or many members of the original one. Even worse, the two committees may be disjoint : this is the Leaving Member Paradox(LMP) ${ }^{25}$. The formal definition follows.

Definition 3. (Leaving Member Paradox) For $m \geq 4$ and $2 \leq g \leq m-1$, the Leaving Member Paradox occurs if for $C^{*}, \hat{C}^{*} \in C^{g}, \exists a \in C^{*}$ and a leaving member $d \in C^{*} \backslash\{a\}$ such that $a \notin \hat{C}^{*}$.

Since we have taken $2 \leq g \leq m-1$, it is clear that with three candidates, we can only elect a committee of size two; so, if a member leaves, there is no reason for the Leaving Member Paradox to happen. The LMP is not defined with less than four candidates.

Recall that the LMP follows the recommendation of rule $R_{2}$; so by following the recommendation of rule $R_{2}$, we can end with a committee totaly different from what we will have with rule $R_{1}$. If a new ballot is taken when an elected candidate leaves, we denote by $P_{\mathrm{LMP}_{j}}^{I C_{\infty}}\left(w, w^{B}\right)$ the limit probability under IC of the LMP given that the leaving candidate is the one who was ranked $j$-th $(j \leq g)$ in the collective ranking. Given that the collective ranking is $d a b c$ and that the two-member committee is $(a, d)$, if candidate $d$ leaves, the $\mathrm{LMP}_{1}$ will occur if the new elected two-member committee is $(b, c)$ i.e the new collective ranking is $b c a$ or $c b a$. So,

$$
P_{\mathrm{LMP}_{1}}^{I C_{\infty}}\left(w, w^{B}\right)=P_{\infty}^{I C}\left(\left(w, w^{B}\right), b c a / d a b c\right)+P_{\infty} I C\left(\left(w, w^{B}\right), c b a / d a b c\right)
$$

\footnotetext{
${ }^{23}$ We admit that, with our computation techniques we are not yet able to get these joint probabilities.

${ }^{24}$ This defines the Limited Voting.

${ }^{25}$ The other paradox described by Staring (1986) in his paper is the Increasing Committee Size Paradox which occurs if an elected member of a committee of size $g$ is no more elected for a committee of size $g+1$; even worse, both committees may be totally disjoint. For more about the Increasing Committee Size Paradox, see Staring (1986), Mitchell and Trumbull (1992), Kamwa (2013).
} 
If candidate $a$ leaves, the $\mathrm{LMP}_{2}$ will occur if the new elected two-member committee is $(d, c)$ i.e the new collective ranking is $b c d$ or $c b d$. So,

$$
\begin{aligned}
P_{\mathrm{LMP}_{2}}^{I C_{\infty}}\left(w, w^{B}\right) & =P_{\infty}^{I C}\left(\left(w, w^{B}\right), b c d / d a b c\right)+P_{\infty}^{I C}\left(\left(w, w^{B}\right), c b d / d a b c\right) \\
& =P_{\infty}^{I C}\left(\left(w, w^{B}\right), b c a / a d b c\right)+P_{\infty}^{I C}\left(\left(w, w^{B}\right), c b a / a d b c\right)
\end{aligned}
$$

$P_{\mathrm{LMP}_{j}}^{I C_{\infty}}\left(w, w^{B}\right)$ are sums of probabilities of inconsistency, it follows directly from Theorem 1 that there are minimized by the Borda rule and maximized by the Plurality rule-Antiplurality rule combination. According to our general results, $P_{\infty}^{I C}\left(\left(w, w^{B}\right), b c a / a d b c\right)<P_{\infty}^{I C}\left(\left(w, w^{B}\right), b c a / d a b c\right)$ and $P_{\infty}^{I C}\left(\left(w, w^{B}\right), c b a / a d b c\right)<P_{\infty}^{I C}\left(\left(w, w^{B}\right), c b a / d a b c\right)$. So, we always get $P_{\mathrm{LMP}_{2}}^{I C_{\infty}}\left(w, w^{B}\right)<P_{\mathrm{LMP}_{1}}^{I C_{\infty}}\left(w, w^{B}\right)$ which means that the $\mathrm{PSP}_{2}$ is always less likely than the $\mathrm{PSP}_{1}$. Thus, in four candidate-election and for two-member committees, the LMP is more likely to occur if the candidate with the highest score in the committee leaves.

Table 10: Likelihood of the $\mathrm{LMP}_{1}$ and the $\mathrm{LMP}_{2}$

\begin{tabular}{||c||c|c||}
\cline { 2 - 3 } \multicolumn{1}{c|}{} & \multicolumn{2}{c|}{ New ballot } \\
\cline { 2 - 3 } \multicolumn{1}{c|}{} & \multicolumn{2}{c|}{$L M P$} \\
\cline { 2 - 3 } & $P_{\mathrm{LMP}_{1}}^{I C_{\infty}}$ & $P_{\mathrm{LMP}_{2}}^{I \mathrm{~L}_{\infty}}$ \\
\hline \hline Borda & 0.018724 & 0.002266 \\
\hline \hline Plurality & \multirow{2}{*}{0.068076} & 0.018987 \\
\hline Antiplurality & & \\
\hline \hline Limited Voting & 0.114152 & 0.046825 \\
\hline \hline
\end{tabular}

For four-candidate elections and two-member committees, Table 10 gives the values of $P_{\mathrm{LMP}_{1}}^{I C_{\infty}}\left(w, w^{B}\right)$ (and that of $P_{\mathrm{LMP}_{2}}^{I C_{\infty}}\left(w, w^{B}\right)$ ) which is the probability of the LMP when the leaving candidate is the one with the highest ( resp. second best) score in the elected two-member committee. According to these probabilities, the Limited voting appears to be the more vulnerable to both the $P_{\mathrm{LMP}_{1}}^{I C}$ and the $P_{\mathrm{LMP}_{2}}^{I C}$ (with respectively $11.41 \%$ and $4.68 \%$ ) than the Plurality rule and the Antiplurality rule $(6.81 \%$ and $1.89 \%)$. The Borda rule minimizes these paradoxes with respectively $1.87 \%$ and $0.23 \%$. For the same reasons we gave for the PSP, our probabilities do not allow us to derive the probability of the LMP no matter the ranking of the leaving candidate.

\section{Conclusion}

The main objective of this paper was to revisit a classical theme in social choice theory, the stability of the collective ranking as candidates leave or enter the choice set. We focussed on the analysis of the discrepancies between the rankings on four-candidate sets and three-candidate sets obtained by scoring rules, by deriving the exact values of the probabilities of all the possible scenarios under the Impartial Culture assumption. We provided results not only for classical rules, such as the Borda count, the simple Plurality rule, and the Antiplurality rule, but also for less known rules, such as the Limited Voting. Our results complete previous analysis by Gehrlein and Fishburn (1980) on the consistency of the rankings obtained by scoring rules on subsets of candidates. As a by product, we could easily estimate from our tables the likelihood of some paradoxical events for the election of a two-member committee from a four candidate menu. We 
showed that the different ways to fill an empty set after an elected member leaves (by picking the prior successor, organizing a partial ballot or starting the whole electoral process again) could lead to very different choices quite frequently for some rules.

Our study confirms the superiority of the Borda count within the class of scoring rules under the Impartial Culture assumption when it comes to minimize the likelihood of discrepancies across subsets of candidates. This fact was already well documented for pairwise majorities; we now extend this result for relationships between four-candidate and three-candidate rankings. Moving up to four candidates also permits us to study rules for which only few things are known, like the Limited Voting. It turns out that it performs quite badly comparatively to the Plurality rule and the Antiplurality rule . In particular, it is unable to keep a ranking in a three candidate subset in more than $50 \%$ of the cases (see Table 6) while the other five scoring rules we study do better, up to $78 \%$ for the Borda count. However, we also observe that obtaining a clear cut ranking of the scoring rules on their propensity to respect orderings is not possible: the Limited Voting does better than Plurality and Antiplurality when it comes to satisfy pairwise rankings. According to Gehrlein and Fishburn (1981), One other thing that can be added to the credit of "Limited voting rules" is that, when one wants to peaks one candidate among many and that each voter vote for his $k$ most preferred candidates, these rules tends to be Condorcet efficient ${ }^{26}$ when $k=\frac{m}{2}$.

When it comes to the selection of a committee, our study compared three ways to fill the empty seat : selecting the prior successor, running a partial election, or starting the electoral process all over again. Though filling the empty seat with the successor is the easiest and costliest method, the figures we observe for the Prior Successor Paradox tell us it may lead us to a wrong choice quite frequently. This paradox is especially large if we use the Plurality rule or the Antiplurality rule: the prior successor could be defeated in almost $32 \%$ of the case in a partial ballot, and in more than $20 \%$ of the case with a full slate election. This figures suggest that, in many cases, it would be a better option to vote again in order to stick to the wishes of the voters. The values we derived for the Leaving Member Paradox teach us that, electing a completely new committee after the departure of a seating member remains a rare event.

At this stage, one may wonder why we did not estimate the discrepancies between the partial ballot method and the full ballot method. To obtain these figures, one needs to estimate the likelihoods of words on, let's say, $\{a, b, c, d\},\{a, b, c\}$ and $\{b, c\}$. To describe a precise word on these subsets, one needs at least six inequalities. Unfortunately, there does not exist general techniques that enable us to derive the exact formulas of the likelihood of such an event under IC. For this reason too, we had to distinguish between $\mathrm{LMP}_{1}$ and $\mathrm{LMP}_{2}$, as we cannot evaluate the likelihood of the joint event. Similarly, we had to distinguish between $\mathrm{PSP}_{1}$ and $\mathrm{PSP}_{2}$ with the new ballot approach. At the moment, one should rely on Monte-Carlo simulations to obtain an estimation of the likelihood of these events.

Hence, though we had a glance at the relationships between the rankings on size three and size four subsets, and at the probabilities of some related events, there are still many question to analyze when we consider more alternatives. As said before, deriving precise formulas for the likelihood of events described by more than five inequalities is almost impossible. Though one may conjecture that the Borda count will minimize the likelihood of inconsistencies on subsets for any number of candidates, a precise proof of this statement is beyond reach. Having more alternatives opens the door to the use of new voting rules. To conclude, only massive simulations

\footnotetext{
${ }^{26} \mathrm{~A}$ voting rule is Condorcet efficient if it always selects the Condorcet winner when he exists.
} 
and/or the development of new computation techniques will enable us to precisely estimate the likelihood of many paradoxes when more than four candidates are in play.

Acknowledgement. We thanks two anonymous reviewers for all their valuable comments we took into account in order to improve the paper. We are particularly grateful to Bernard Grofman for his useful comments. We would also like to thank seminar audience at the $12^{\text {th }}$ Meeting of the Society for Social Choice and Welfare (Boston), at the 2014 Political Economy Workshop (Milan), at the 2014 Summer School "Interdisciplinary Analysis of Voting Rules" COST Action IC1205 (Caen), and at the Journée Doctorale-EDEHN ”Analyse des Politiques Publiques” (Université du Havre).

\section{References}

Berg, S., Lepelley, D., 1994. On probability models in voting theory. Statistica Neerlandica 48, 133-146.

J.C. de Borda. Mémoire sur les élections au scrutin. Histoire de l'Académie Royale des Sciences, Paris, 1781.

Cervone, D., Gehrlein, W.V., Zwicker, W., 2005. Which scoring rule maximizes Condorcet efficiency under IAC? Theory and Decision 58(2), 145-185.

Chevaleyre, Y., Lang, J., Maudet, N., Monnot, J., Xia, L. 2012. New candidates welcome! Possible winners with respect to the addition of new candidates. Mathematical Social Sciences, 64(1),74-88.

Marquis de Condorcet, 1785. Essai sur l'application de l'analyse à la probabilité des décisions rendues à la pluralité des voix. Paris.

Coxeter, H.S.M., 1935. The functions of Schläfli and Lobatschefsky. Quarterly Journal of Mathematics 6, 13-29.

Diss, M., Merlin, V., Valognes, F., 2010. On the Condorcet efficiency of approval voting and extended Scoring Rules for Three Alternatives. In Hanbook of Approval Voting, Laslier J-F. and Sanver R. (Eds), Studies in Choice and Welfare Springer Berlin Heidelberg.

Dodgson, C.L., 1876. A method of taking Votes on more than two Issues. Clarendon Press, Oxford.

Dummett, M., 1984. Voting procedures. Oxford, Clarendon Press.

Dutta, B., Jackson, M.O., Le Breton, M., 2001. Strategic candidacy and voting procedures. Econometrica 69(4), 10131037.

Felsenthal, D., 2012. Review of paradoxes afflicting procedures for electing a single candidate. In Electoral Systems : Paradoxes, Assumptions, and Procedures, Felsenthal, D. \& Machover, M. (Eds.), Studies in Choice and Welfare Springer Berlin Heidelberg.

Fishburn, P.C., 1981. Inverted orders for monotone scoring rules. Discrete Applied Mathematics 3, 27-36.

Fishburn, P.C., Gehrlein W.V., 1976. Borda's rule, positional voting and Condorcet's simple majority principle. Public Choice 28, 79-88.

Gehrlein, W.V., Fishburn, P.C., 1976. The probability of the paradox of voting: A computable solution. Journal of Economic Theory 13, 14-25.

Gehrlein W.V., Fishburn P.C., 1980. Robustness of positional scoring over subsets of alternatives. Appl. Math. Optim. 6, 241-255.

Gehrlein W.V., Fishburn, P.C., 1981. Scoring rules and majority agreements for large electorates with arbitrary preferences. Mathematical Social Sciences 2, 23-33.

Gehrlein W.V., Fishburn, P.C., 1981. Constant scoring rules for choosing one among many alternatives. Quality and Quantity 15, 203-210.

Gehrlein, W.V., Gopinath, B., Lagarias J.C., Fishburn P.C., 1982. Optimal pairs of score vectors for positional scoring rules. Appl. Math. Optim. 8, 309-324.

Gehrlein, W.V., Lepelley D., 2010. Voting paradoxes and group coherence. Springer.

Kamwa, E., 2013. The increasing committee size paradox with a small number of candidates. Economics Bulletin, 33(2), 967-972.

Kamwa, E., Merlin V., 2015. Scoring Rules over Subsets of Alternatives : A General Formula to Analyze Consistency in Four-candidate Elections Under the Impartial Culture. Available online here.

Kellerhals, R., 1989. On the volume of hyperbolic polyedra. Mathematische Annalen 285, 541-569.

Lang, J., Maudet, N., Polukarov, M., 2013. New results on equilibria in strategic candidacy. Algorithmic Game Theory Lecture Notes in Computer Science 8146, 13-25

McLean, I., Urken A., 1995. Classics of social Choice. Michigan University Press. 
Merlin, V., Tataru, M., Valognes, F., 2000. On the probability that all decision rules select the same winner. Journal of Mathematical Economics 33, 183-207.

Merlin, V., Valognes, F., 2004. On the impact of indifferent voters on the likelihood of some voting paradoxes. Mathematical Social Sciences 48, 343-361.

Milnor, J., 1982. Hyperbolic geometry: the first 150 years. Bull AMS 6, 9-24.

Mitchell, D.W., Trumbull, W.N., 1992. Frequency of paradox in a common N-winner voting scheme. Public Choice 73(1), 55-69.

Nanson, E.J., 1882. Methods of election. Transactions and Proceedings of Royal Society of Victoria 19, 197-240.

Saari, D.G., 1987. The source of some paradoxes from social choice and statistics. Journal of Econ. Theory 41, 1-22.

Saari, D.G., 1988. A dictionary for voting paradoxes. Journal of Economic Theory 48, 443-475.

Saari, D.G., 1990. The Borda dictionary. Social Choice and Welfare 7, 279-317.

Saari, D.G., 1996. Election relations and a partial Ordering for positional voting. In Collective Decision-Making: Social Choice and Political Economy, Schofield N. (Ed.), Springer, 993-110.

Saari, D.G., Merlin, V., 1996. The Copeland method I. Relationships and the dictionary. Economic Theory 8-1, 51-76.

Saari, D.G., Tataru, M., 1999. The likelihood of dubious election outcomes. Economic Theory 13, 345-363.

Schläfli, L., 1950. Theorie der vielfachen kontinuität. Gesammelte Mathematische Abhandlungen 1. Birkhäuser, Basel.

Smith, J., 1973. Aggregation of preferences with variable electorate. Econometrica 41, 1027-1041

Staring, M., 1986. Two paradoxes of committee elections. Mathematics Magazine 59(3), 158-159.

Tataru, M., Merlin, V., 1997. On the relationship of the Condorcet winner and positional voting rules. Mathematical Social Sciences 34, 81-90.

Tideman, T.N., 1987. Independence of clones as a criterion for voting Rules. Social Choice and Welfare 4, 185-206.

Van Newenhizen, J., 1992. The Borda method is most likely to respect the Condorcet principle. Economic Theory 2, 69-83.

Young H.P. (1988) Condorcet's theory of voting. American Political Science Review 82-4: 1231-1244. 